\title{
Quaternion-Based Attitude Control System Design of Single and Cooperative Spacecrafts: Boundedness of Solution Approach
}

\author{
Harry Septanto, ${ }^{1,2}$ Riyanto T. Bambang, ${ }^{1}$ Arief Syaichu-Rohman, \\ Ridanto Eko Poetro, ${ }^{3}$ and Adrianto Ravi Ibrahim ${ }^{1}$ \\ ${ }^{1}$ School of Electrical Engineering and Informatics, Institut Teknologi Bandung, Bandung 40123, Indonesia \\ ${ }^{2}$ Center for Satellite Technology, National Institute of Aeronautics and Space (LAPAN), Bogor 16310, Indonesia \\ ${ }^{3}$ Faculty of Mechanical and Aerospace Engineering, Institut Teknologi Bandung, Bandung 40123, Indonesia
}

Correspondence should be addressed to Harry Septanto; hseptanto@gmail.com

Received 25 December 2013; Accepted 21 April 2014; Published 19 May 2014

Academic Editor: Franco Ramírez

Copyright (C) 2014 Harry Septanto et al. This is an open access article distributed under the Creative Commons Attribution License, which permits unrestricted use, distribution, and reproduction in any medium, provided the original work is properly cited.

\begin{abstract}
It is well known that single equilibrium orientation point in matrix rotation is represented by two equilibrium points in quaternion. This fact would imply nonefficient control effort as well as problem in guaranteeing stability of the two equilibrium points in quaternion. This paper presents a solution to design quaternion-based spacecraft attitude control system whose saturation element is in its control law such that those problems are overcome. The proposed feature of methodology is the consideration on boundedness of solution in the control system design even in the presence of unknown external disturbance. The same methodology is also used to design cooperative spacecrafts attitude control system. Through the proposed method, the most relaxed information-state topology requirement is obtained, that is, the directed graph that contains a directed spanning tree. Some numerical simulations demonstrate effectiveness of the proposed feature of methodology.
\end{abstract}

\section{Introduction}

Over the past decades, spacecraft developments have been done to bring wide-range missions, including earth observation and communication. Attitude control system plays an important role in these missions. Many research efforts on spacecraft attitude control design have been reported, that is, linear matrix inequality- (LMI-) based robust mixed $\mathrm{H}_{2} / \mathrm{H}_{\mathrm{\infty}}$ attitude control system design with linearization of dynamics and kinematics approach [1]; LMI-based nonlinear continuous attitude control design, [2]; proportional-derivative+ $(\mathrm{PD}+)$ type output feedback attitude control system with uniformly practically asymptotically stability guarantee for its equilibrium points [3]; attitude control system with discontinuous control law applying inverse cotangent function [4]; and hybrid attitude control system with property of robustness to measurement noise [5], to name a few.

From an attitude determination calculation, orientation of spacecraft is obtained as a rotation matrix that belongs to special orthogonal order-3 space, $\mathrm{SO}(3)$. However, some parameterizations are usually employed in a design of attitude control that is also employed in [1-5] —all of them employ quaternion parameterization, except [1], which uses Euler angle parameterization.

Because quaternion can represent spacecraft attitude globally, it is useful for spacecraft whose missions require doing reorientation maneuver over a large rotation angle. In contrast, Euler angle parameterization cannot represent spacecraft attitude globally due to its singularity property. Note that quaternion-also called Euler parameters-is the only parameterization consisting of four parameters and can represent attitude or orientation globally [6]. Nevertheless, its representation is not unique. There are two-antipodal values that correspond to a single physical orientation or a single orientation in matrix rotation representation. Therefore, single equilibrium orientation point in matrix rotation representation is represented by two equilibrium points in quaternion. This fact would imply nonefficient control effort called 
unwinding phenomenon when only one equilibrium point is regarded in quaternion-based attitude control design [7]. Besides, difficulty in guaranteeing stability appeared when the two equilibrium points in quaternion are regarded. There are many research results giving solution to this problem. They include discontinuous control approach [4], optimal control and finite time stability approach [8], backsteppingbased attitude control design [9], hybrid mechanism attitude control $[5,10]$, and continuous nonlinear attitude control employing augmented dynamic [11], to name a few.

In addition to above problem, numerous spacecraft formation flying missions have been conceived and some of them have been launched, for example, interferometric synthetic aperture radar spacecrafts [12] and rendezvous technology experiment [13]. Cooperative control is a main issue for spacecraft formation control. In [14], Ren proposed proportional-derivative (PD) type attitude controller with several consensus algorithms for attitude formation. Hybrid attitude controller with connected and acyclic exchange of information-state topology for attitude formation has been reported in [15]. In [16], PD type controller with connected (undirected) exchange of information-state topology for attitude formation has been designed through Input to state stability approach where cooperative controller term is regarded as external input of each spacecraft.

In this paper, attitude controller appearing in [17] is utilized. Main contributions of this paper are attitude control system designed through boundedness of solution approach, that is, ultimately bounded solution and input to state stability for single attitude control case and boundedness of solution for cooperative attitude control case. Unlike the attitude control system employing PD controller that considers only one equilibrium point in quaternion representation, the attitude controller used in this paper considers two equilibrium points in quaternion representation. Less strict information-state topology requirement for attitude formation is also obtained, that is, topology that consists of a directed spanning tree. Preliminary result of this research has been presented in [18].

Overall, this paper is organized as follows. Section 2 discusses the system model. This section consists of notation, kinematics, and dynamics model of spacecraft and information-state exchange framework of the cooperative spacecrafts used in the rest of the paper. Section 3 describes controllers design and simulations. Finally, some concluding remarks are stated in Section 4.

\section{Modeling}

In this paper, a spacecraft is considered a rigid body. In the following descriptions, subscripts or/and superscripts $b, l$, and $d$ denote spacecraft's fixed body frame, inertial reference frame, and spacecraft's desired frame, respectively. For brevity, the spacecraft's fixed body frame, the inertial reference frame, and the spacecraft's desired frame may be written as body frame, inertial frame, and desired frame, respectively. Here, set of $n$-dimension real column matrices, set of $n \times m$ real matrices, and set of positive integers are denoted by $\mathfrak{R}^{n}, \mathfrak{R}^{n \times m}$, and $Z_{>0}$, respectively, where $n, m \in$
$Z_{>0}$. Given $y \in \Re^{n}$ and $Y \in \mathfrak{R}^{n \times n},\|y\|$ is 2 -norm of column matrix $y ; \lambda_{\min }(Y)$ and $\lambda_{\max }(Y)$ are minimum and maximum Eigen value of $Y$, respectively; $y^{T}$ and $Y^{T}$ are transpose matrix of $y$ and $Y$, respectively; $Y>0$ means that $Y$ is a positive definite matrix.

Following [19], vectors are defined as follows:

$$
\vec{r}=r^{b^{T}} \mathrm{~F}_{b}
$$

where $F_{b}$ is a vectrix associated to the body frame, that is, a column matrix with three unit vectors $\widehat{l}_{1}, \widehat{l}_{2}$, and $\widehat{l}_{3}-\mathrm{F}_{l}=$ $\left[\begin{array}{lll}\widehat{l}_{1} & \widehat{l}_{2} & \widehat{l}_{3}\end{array}\right]^{T} ; r^{b} \in \mathfrak{R}^{3}$ is a column matrix whose three components of $\vec{r}$ are expressed or decomposed into the body frame; ${ }_{\mu} r^{b}$ is skew-symmetric matrix of $r^{b}$.

2.1. Spacecraft Kinematics and Dynamics Model. Consider the rotation matrix $R_{b d}$ to transform a vector expressed in $\mathrm{F}_{b}$ to be expressed in $\mathrm{F}_{d}$ that satisfies (2):

$$
R_{b d}=\mathrm{F}_{b} \cdot \mathrm{F}_{d}^{T} \Longleftrightarrow \mathrm{F}_{d}^{T}=\mathrm{F}_{b}^{T} R_{b d} .
$$

Taking the time derivative of (2) w.r.t. (with respect to) $F_{d}$, $0=\mathrm{F}_{b}^{T}\left({ }_{\mu} \omega_{b d}^{b} R_{b d}+\dot{R}_{b d}\right)$ is satisfied. Moreover, it implies (3), that is, the $\mathrm{SO}(3)$ rotation matrix based kinematics equation of body frame w.r.t. desired frame:

$$
\dot{R}_{b d}=-{ }_{\mu} \omega_{b d}^{b} R_{b d} .
$$

The corresponding quaternion-based kinematics equation of body frame w.r.t. desired frame is given by

$$
\dot{q}_{b d}=\left[\begin{array}{ll}
\dot{\eta}_{b d} & \dot{\varepsilon}_{b d}^{T}
\end{array}\right]^{T},
$$

where

$$
\begin{gathered}
\dot{\eta}_{b d}=-\frac{1}{2} \varepsilon_{b d}^{T} \omega_{b d}^{b}, \\
\dot{\varepsilon}_{b d}=\frac{1}{2}\left({ }_{\mu} \varepsilon_{b d}+\eta_{b d} I\right) \omega_{b d}^{b} .
\end{gathered}
$$

Since quaternion is member of unit sphere order $-3, S^{3}, q_{b d}=$ $\left[\begin{array}{ll}\eta_{b d} & \varepsilon_{b d}^{T}\end{array}\right]^{T}$ satisfies $\left\|q_{b d}\right\|^{2}=1$, where $\eta_{b d}=\cos \left(\phi_{b d} / 2\right) \in \Re$ and $\varepsilon_{b d}=\widehat{a}_{b d} \sin \left(\phi_{b d} / 2\right) \in \mathfrak{R}^{3} ; \widehat{a}_{b d}$ is the corresponding Euler axis and $\phi_{b d}$ is the corresponding Euler angle. Note that, here, this kinematics also represents attitude error.

Dynamic of the rigid body rotation system decomposed in $F_{b}$ is given by the Euler equation [19] (6):

$$
J \dot{\omega}_{b l}^{b}=-{ }_{\mu} \omega_{b l}^{b} J \omega_{b l}^{b}+\tau+d,
$$

where positive definite matrix $J \in \mathfrak{R}^{3 \times 3}$ is a spacecraft moment of inertia about its center of mass located in the origin of $\mathrm{F}_{b}\left(\mathrm{~kg} \mathrm{~m}^{2}\right) ; \omega_{b l}^{b} \in \mathfrak{R}^{3}$ is angular velocity of $\mathrm{F}_{b}$ w.r.t. $\mathrm{F}_{l}$ decomposed in $\mathrm{F}_{b},(\mathrm{rad} / \mathrm{s}) ; \tau \in \mathfrak{R}^{3}$ is the total external control torque about its center of mass located in the origin of $\mathrm{F}_{b}(\mathrm{Nm})$; and $d \in \mathfrak{R}^{3}$ is the external disturbance torque or total environmental effects, for example, gravity-gradient effects, solar radiation, magnetic field torques, and air-drag. 
2.2. Cooperative Attitude Framework. Index of a spacecraft in a group composed by $n$-spacecrafts is denoted by subscript (and sometimes both subscript and superscript) ( $i$ ) and its neighbor is denoted by subscript (and sometimes both subscript and superscript) $(j)$, where $1 \leq i, j \leq n \in Z_{>0}$. For example, $\mathrm{F}_{b(i)}$ denotes body frame of spacecraft $i$.

The dynamic of spacecraft $i$ in a group is represented by

$$
J_{(i)} \dot{\omega}_{b(i) l}^{b(i)}=-{ }_{\mu} \omega_{b(i) l}^{b(i)} J_{(i)} \omega_{b(i) l}^{b(i)}+\tau_{(i)}+d_{(i)}
$$

Quaternion-based kinematics equation that represents orientation of body frame of spacecraft $i, \mathrm{~F}_{b(i)}$, w.r.t. body frame of spacecraft $j, \mathrm{~F}_{b(j)}$, is

$$
\dot{q}_{b(i) b(j)}=\left[\begin{array}{ll}
\dot{\eta}_{b(i) b(j)} & \dot{\varepsilon}_{b(i) b(j)}^{T}
\end{array}\right]^{T}
$$

where

$$
\begin{gathered}
\dot{\eta}_{b(i) b(j)}=-\frac{1}{2} \varepsilon_{b(i) b(j)}^{T} \omega_{b(i) b(j)}^{b(i)}, \\
\dot{\varepsilon}_{b(i) b(j)}=\frac{1}{2}\left({ }_{\mu} \varepsilon_{b(i) b(j)}+\eta_{b(i) b(j)} I\right) \omega_{b(i) b(j)}^{b(i)} .
\end{gathered}
$$

Information-state exchange between spacecraft in a group composed by $n$-spacecraft is modeled by a directed graph topology

$$
\mathrm{G}_{n} \equiv\left(\mathrm{V}_{n}, \mathrm{E}_{n}, \mathrm{~A}_{n}\right)
$$

where $\mathrm{V}_{n}=\left\{v_{(i)}\right\}$ is the node set; $\mathrm{E}_{n} \subseteq \mathrm{V}_{n} \times \mathrm{V}_{n}$ is the edges set; and $A_{n}=\left[a_{(i)(j)}\right] \in \mathfrak{R}^{n \times n}$ is the adjacency matrix of the graph $\mathrm{G}_{n}$, where $1 \leq i, j \leq n \in Z_{>0}$.

The entry of adjacency matrix $a_{(i)(j)}=1$ if spacecraft $i$ receives information-state from spacecraft $j$, where $v_{(j)}$ is the parent node and $v_{(i)}$ is child node and $\left(v_{(i)} v_{(j)}\right) \in \mathrm{E}_{n}$. If there is no information-state exchange from spacecraft $j$ to spacecraft $i$, then $a_{(i)(j)}=0$. Self-edge is not allowed, that is, $a_{(i)(i)}=0$. In a directed graph, $a_{(i)(j)} \neq a_{(j)(i)}$. If the graph $G_{n}$ has at least one node with a directed path to all other nodes then $\mathrm{G}_{n}$ is called to have a directed spanning tree.

\section{Control Systems Design and Simulations}

Equations (12) and (13) [17] are the feedback term for (6) and (7), respectively, where $k \in \Re$ and $L \in \mathfrak{R}^{3 \times 3}$ are tuning parameters. The function $\Phi_{\varepsilon_{b d}}$ is a column matrix of saturation function that, defined element-wise, follows the scalar saturation function $\phi_{x}(14)$, with the saturation limit $0<\bar{\phi}<\sqrt{1 / 3} \in \mathfrak{R}$ :

$$
\begin{aligned}
\tau & =-k\left(\eta_{b d} \varepsilon_{b d}+\Phi_{\varepsilon_{b d}}-\varepsilon_{b d}\right)-L \omega_{b l}^{b} \\
& =-k\left(\eta_{b d} \varepsilon_{b d}-\Psi_{\varepsilon_{b d}}\right)-L \omega_{b l}^{b},
\end{aligned}
$$

$$
\begin{array}{r}
\tau_{(i)}=-\sum_{j=1}^{n} a_{(i)(j)}\left(k\left(\eta_{b(i) b(j)} \varepsilon_{b(i) b(j)}+\Phi_{\varepsilon_{b(i) d(j)}}-\varepsilon_{b(i) d(j)}\right)\right. \\
\left.+L \omega_{b(i) l}^{b(i) l} k\right) \\
=-\sum_{j=1}^{n} a_{(i)(j)}\left(k\left(\eta_{b(i) b(j)} \varepsilon_{b(i) b(j)}-\Psi_{\varepsilon_{b(i) d(j)}}\right)+L \omega_{b(i) l}^{b(i) l}\right),
\end{array}
$$

$$
\phi_{x}= \begin{cases}\bar{\phi}, & x>\bar{\phi} \\ x, & -\bar{\phi} \leq x \leq \bar{\phi} \\ -\bar{\phi}, & x<-\bar{\phi}\end{cases}
$$

3.1. Problem Statements of the Control Systems Design. Next subsection would discuss the proposed attitude control design for single spacecraft case as well as cooperative spacecraft case. By utilizing Lyapunov stability theory, $k \in \Re$ and $L \in \Re^{3 \times 3}$ have to be found such that the solution of single spacecraft attitude control system composed by (6) and (12) is ultimately bounded if $d=0$ and is input to state stable if $d \neq 0$. Similarly, a cooperative spacecraft attitude control has to be designed by determining $k \in \mathfrak{R}$ and $L \in \mathfrak{R}^{3 \times 3}$ and information-state exchange topology such that the solution of each spacecraft in a group is ultimately bounded if $d=$ 0 and is input to state stable if $d \neq 0$. The authors suggest the readers should refer to [20] for definition of ultimately bounded solution and input to state stability.

3.2. Single Spacecraft Case. It is well known that Lyapunov stability theory requires the existence and uniqueness of a solution, for a given initial condition, for all future time. The following proposition states that the system composed by (6) and (12) satisfies existence and uniqueness solution requirement because the system is locally Lipschitz [19].

Proposition 1. Consider the system (6). Suppose $d=0$. The spacecraft attitude control system composed by (4)-(6) and (12) is locally Lipschitz in $\left(q_{b d}=\left[\begin{array}{ll}\eta_{b d} & \varepsilon_{b d}\end{array}\right]^{T}, \omega_{b l}^{b}\right) \in S^{3} \times \mathfrak{R}^{3}$.

Proof. For brevity, let $\omega_{b l_{p}}^{b}=\omega_{p}, d \omega_{b l_{p}}^{b} / d t=\dot{\omega}_{b l_{p}}^{b}, \varepsilon_{b d_{p}}=\varepsilon_{p}$, and $\eta_{b d_{p}}=\eta_{p}$, where $p=\{1,2\}$. Then, consider the following:

$$
\begin{aligned}
& \left\|\begin{array}{c}
\dot{\eta}_{1}-\dot{\eta}_{2} \\
\dot{\varepsilon}_{1}-\dot{\varepsilon}_{2} \\
J \dot{\omega}_{1}-J \dot{\omega}_{2}
\end{array}\right\| \\
& =\sqrt{\left\|\dot{\eta}_{1}-\dot{\eta}_{2}\right\|^{2}+\left\|\dot{\varepsilon}_{1}-\dot{\varepsilon}_{2}\right\|^{2}+\left\|J \dot{\omega}_{1}-J \dot{\omega}_{2}\right\|^{2}} \\
& \Longleftrightarrow\left\|\begin{array}{c}
\dot{\eta}_{1}-\dot{\eta}_{2} \\
\dot{\varepsilon}_{1}-\dot{\varepsilon}_{2} \\
J \dot{\omega}_{1}-J \dot{\omega}_{2}
\end{array}\right\|^{2} \\
& =\left\|\dot{\eta}_{1}-\dot{\eta}_{2}\right\|^{2}+\left\|\dot{\varepsilon}_{1}-\dot{\varepsilon}_{2}\right\|^{2}+\left\|J \dot{\omega}_{1}-J \dot{\omega}_{2}\right\|^{2} .
\end{aligned}
$$


Since (5) are continuously differentiable, then the following inequalities can be obtained

$$
\begin{gathered}
\left\|\dot{\eta}_{1}-\dot{\eta}_{2}\right\| \leq \ell_{a}\left\|\begin{array}{c}
\varepsilon_{1}-\varepsilon_{2} \\
\omega_{1}-\omega_{2}
\end{array}\right\| \leq \ell_{a}\left\|\begin{array}{c}
\eta_{1}-\eta_{2} \\
\varepsilon_{1}-\varepsilon_{2} \\
\omega_{1}-\omega_{2}
\end{array}\right\| \\
\left\|\dot{\varepsilon}_{1}-\dot{\varepsilon}_{2}\right\| \leq \ell_{b}\left\|\begin{array}{c}
\eta_{1}-\eta_{2} \\
\varepsilon_{1}-\varepsilon_{2} \\
\omega_{1}-\omega_{2}
\end{array}\right\|,
\end{gathered}
$$

where $\ell_{a}, \ell_{b} \in \mathfrak{R}>0$.

Now consider $\left\|J \dot{\omega}_{1}-J \dot{\omega}_{2}\right\|$ as follows:

$$
\begin{aligned}
\left\|J \dot{\omega}_{1}-J \dot{\omega}_{2}\right\|=\| & -{ }_{\mu} \omega_{1} J \omega_{1}-k\left(\eta_{1} \varepsilon_{1}+\Phi_{\varepsilon_{1}}-\varepsilon_{1}\right)-L \omega_{1} \\
& +{ }_{\mu} \omega_{2} J \omega_{2}+k\left(\eta_{2} \varepsilon_{2}+\Phi_{\varepsilon_{2}}-\varepsilon_{2}\right)+L \omega_{2} \| \\
\left\|J \dot{\omega}_{1}-J \dot{\omega}_{2}\right\| \leq & \left\|-{ }_{\mu} \omega_{1} J \omega_{1}+{ }_{\mu} \omega_{2} J \omega_{2}\right\| \\
& +\left\|-k\left(\eta_{1} \varepsilon_{1}-\varepsilon_{1}\right)+k\left(\eta_{2} \varepsilon_{2}-\varepsilon_{2}\right)\right\| \\
& +\left\|-k \Phi_{\varepsilon_{1}}+k \Phi_{\varepsilon_{2}}\right\|+\left\|-L \omega_{1}+L \omega_{2}\right\|
\end{aligned}
$$

Since $f_{a}\left(\omega_{p}\right)=-{ }_{\mu} \omega_{p} J \omega_{p}$ and $f_{b}\left(\eta_{p}, \varepsilon_{p}\right)=-k\left(\eta_{p} \varepsilon_{p}-\varepsilon_{p}\right)$, where $p=\{1,2\}$, are continuously differentiable functions, then the following inequalities can be obtained:

$$
\begin{gathered}
\left\|f_{a}\left(\omega_{1}\right)-f_{a}\left(\omega_{2}\right)\right\| \leq \ell_{c}\left\|\omega_{1}-\omega_{2}\right\| \leq \ell_{c}\left\|\begin{array}{c}
\eta_{1}-\eta_{2} \\
\varepsilon_{1}-\varepsilon_{2} \\
\omega_{1}-\omega_{2}
\end{array}\right\| \\
\left\|f_{b}\left(\omega_{1}\right)-f_{b}\left(\omega_{2}\right)\right\| \leq \ell_{d}\left\|\begin{array}{c}
\eta_{1}-\eta_{2} \\
\varepsilon_{1}-\varepsilon_{2}
\end{array}\right\| \leq \ell_{d}\left\|\begin{array}{c}
\eta_{1}-\eta_{2} \\
\varepsilon_{1}-\varepsilon_{2} \\
\omega_{1}-\omega_{2}
\end{array}\right\|,
\end{gathered}
$$

where $\ell_{c}, \ell_{d} \in \mathfrak{R}>0$.

Now, recall (18) and (19), since the term with saturation function satisfies $\left\|-k \Phi_{\varepsilon_{1}}+k \Phi_{\varepsilon_{2}}\right\| \leq k\left\|\varepsilon_{1}-\varepsilon_{2}\right\| \leq k\left\|\begin{array}{c}\eta_{1}-\eta_{2} \\ \varepsilon_{1}-\varepsilon_{2} \\ \omega_{1}-\omega_{2}\end{array}\right\|$, where $k>0$, then the following inequalities can be obtained:

$$
\begin{aligned}
& \left\|J \dot{\omega}_{1}-J \dot{\omega}_{2}\right\| \\
& \leq \ell_{c}\left\|\begin{array}{c}
\eta_{1}-\eta_{2} \\
\varepsilon_{1}-\varepsilon_{2} \\
\omega_{1}-\omega_{2}
\end{array}\right\|+\ell_{d}\left\|\begin{array}{c}
\eta_{1}-\eta_{2} \\
\varepsilon_{1}-\varepsilon_{2} \\
\omega_{1}-\omega_{2}
\end{array}\right\|+k\left\|\begin{array}{c}
\eta_{1}-\eta_{2} \\
\varepsilon_{1}-\varepsilon_{2} \\
\omega_{1}-\omega_{2}
\end{array}\right\| \\
& +\lambda_{\max }(L)\left\|\begin{array}{c}
\eta_{1}-\eta_{2} \\
\varepsilon_{1}-\varepsilon_{2} \\
\omega_{1}-\omega_{2}
\end{array}\right\| \\
& \leq\left(\ell_{c}+\ell_{d}+k+\lambda_{\max }(L)\right)\left\|\begin{array}{c}
\eta_{1}-\eta_{2} \\
\varepsilon_{1}-\varepsilon_{2} \\
\omega_{1}-\omega_{2}
\end{array}\right\|,
\end{aligned}
$$

where symmetric matrix $L>0$.
Recall (15), (16), and (20), then the following inequalities can be obtained:

$$
\begin{aligned}
& \left\|\begin{array}{c}
\dot{\eta}_{1}-\dot{\eta}_{2} \\
\dot{\varepsilon}_{1}-\dot{\varepsilon}_{2} \\
J \dot{\omega}_{1}-J \dot{\omega}_{2}
\end{array}\right\|^{2} \\
& \leq\left(\ell_{a}^{2}+\ell_{b}^{2}+\left(\ell_{c}+\ell_{d}+k+\lambda_{\max }(L)\right)^{2}\right)\left\|\begin{array}{c}
\eta_{1}-\eta_{2} \\
\varepsilon_{1}-\varepsilon_{2} \\
\omega_{1}-\omega_{2}
\end{array}\right\|^{2} \\
& \Longleftrightarrow\left\|\begin{array}{c}
\dot{\eta}_{1}-\dot{\eta}_{2} \\
\dot{\varepsilon}_{1}-\dot{\varepsilon}_{2} \\
J \dot{\omega}_{1}-J \dot{\omega}_{2}
\end{array}\right\| \\
& \leq \sqrt{\ell_{a}^{2}+\ell_{b}^{2}+\left(\ell_{c}+\ell_{d}+k+\lambda_{\max }(L)\right)^{2}}\left\|\begin{array}{c}
\eta_{1}-\eta_{2} \\
\varepsilon_{1}-\varepsilon_{2} \\
\omega_{1}-\omega_{2}
\end{array}\right\|,
\end{aligned}
$$

where $\ell_{a}, \ell_{b}, \ell_{c}, \ell_{d}, k>0$ and symmetric matrix $L>0$.

The last inequality in (21) states that the system composed by (4)-(6) and (12) is locally Lipschitz in $\left(q_{b d}=\right.$ $\left.\left[\begin{array}{ll}\eta_{b d} & \varepsilon_{b d}\end{array}\right]^{T}, \omega_{b l}^{b}\right) \in S^{3} \times \mathfrak{R}^{3}$. It implies that the system has a unique solution for all future time.

Proposition 2. Consider the system (6). If $d$ is continuous and bounded for all $t \geq 0$, then the attitude control system composed by (4)-(6) and (12) satisfies local Lipschitz in $\left(q_{b d}=\right.$ $\left.\left[\begin{array}{ll}\eta_{b d} & \varepsilon_{b d}\end{array}\right]^{T}, \omega_{b l}^{b}, d\right) \in S^{3} \times \mathfrak{R}^{3} \times \mathfrak{R}^{3}$.

Proof. Follow idea of the proof of Proposition 1.

Theorem 3. Consider the system (6) and suppose there is no external disturbance, that is, $d=0$. If scalar $k$ is positive, matrix $L$ and moment of inertia $J$ are symmetric and positive definite, then solution of the quaternion-based attitude control system composed by (4)-(6) and (12) is ultimately bounded.

Proof. Consider S, a set that consists of all $\varepsilon_{b d},(22)$, and positive definite function (23):

$$
\begin{gathered}
S=\left\{\varepsilon_{b d} \in \mathfrak{R}^{3}:-1 \leq \eta_{b d} \leq 1 \in \Re, \eta_{b d}^{2}+\varepsilon_{b d}^{T} \varepsilon_{b d}=1\right\} \\
V=k \varepsilon_{b d}^{T} \varepsilon_{b d}+\frac{1}{2} \omega_{b l}^{b^{T}} J \omega_{b l}^{b}=\left[\begin{array}{c}
\varepsilon_{b d} \\
b \\
\omega_{b l}^{b}
\end{array}\right]^{T} P\left[\begin{array}{c}
\varepsilon_{b d} \\
b \\
\omega_{b l}^{b}
\end{array}\right],
\end{gathered}
$$

where

$$
P=\left[\begin{array}{cc}
k I & 0 \\
0 & \frac{1}{2} J
\end{array}\right], \quad I=\left[\begin{array}{lll}
1 & 0 & 0 \\
0 & 1 & 0 \\
0 & 0 & 1
\end{array}\right]
$$

To make sure that $P$ is a positive definite matrix, that is, $P>0$, hence $k>0 \in \Re$ and symmetric matrix $J>0 \in \Re^{3 \times 3}$. If $J$ has three different eigenvalues, then $V$ will be bounded below by $\alpha_{1}$ and bounded above by $\alpha_{2}$, where $\alpha_{1}$ and $\alpha_{2}$ are class $K_{\infty}$ functions given below:

$$
\alpha_{1}=\lambda_{\min }(P)\left\|\begin{array}{c}
\varepsilon_{b d} \\
b \\
\omega_{b l}^{b}
\end{array}\right\|^{2} \leq V \leq \alpha_{2}=\lambda_{\max }(P)\left\|\begin{array}{c}
\varepsilon_{b d} \\
b \\
\omega_{b l}^{b}
\end{array}\right\|^{2} .
$$


Now, take time derivative of (23). Since the desired angular velocity $\omega_{d l}^{b}$ is zero, $\omega_{b d}^{b}=\omega_{b l}^{b}$. In addition, note that $\varepsilon_{b d \mu}^{T} \varepsilon_{b d}=$ $\omega_{b l}^{b^{T}} \omega_{b l}^{b}=0$ (see Fact 3.10.1 in [21]). Following these, (26) is satisfied:

$$
\dot{V}=k \omega_{b l}^{b^{T}} \Psi_{\varepsilon_{b d}}-\omega_{b l}^{b^{T}} L \omega_{b l}^{b}
$$

$V$ is a nondecreasing function since $\dot{V}$ is not always negative. Fortunately, the unit quaternion is bounded by the unit sphere order-3 property (27):

$$
\left\|q_{b d}\right\|^{2}=\eta_{b d}^{2}+\varepsilon_{b d}^{T} \varepsilon_{b d}=1
$$

Therefore, the solution $\varepsilon_{b d}$ is bounded above by 1 as shown below:

$$
\left\|\varepsilon_{b d}(t)\right\| \leq 1, \quad t \geq 0
$$

As a direct consequence of (28), $\Psi_{\varepsilon_{b d}}(t)$ is bounded above by $\kappa_{1}$, that is,

$$
\left\|\Psi_{\varepsilon_{b d}}\right\| \leq \kappa_{1}=\max \left\{3 \cdot\left(\sqrt{\frac{1}{3}}-\bar{\phi}\right)^{2},(1-\bar{\phi})^{2}\right\}<1 .
$$

In addition, for all positive scalar $k$ and positive definite symmetric matrix $L$, if $\dot{V}>0$, then the solution $\omega_{b l}^{b}(t)$ is bounded, that is,

$$
\left\|\omega_{b l}^{b}\right\| \leq \frac{k}{\lambda_{\min }(L)} \kappa_{1} .
$$

Note that $L$ has to be a symmetric and positive definite matrix to make sure that all eigenvalue of $L$ are positive so that, along with $k>0,(30)$ is satisfied.

From (28)-(30), $V$ is bounded as follows:

$$
V \leq k+\frac{1}{2} \lambda_{\max }(J)\left(\frac{k \cdot \kappa_{1}}{\lambda_{\min }(L)}\right)^{2}=\kappa_{2}
$$

Consider (26); using property (29), the following inequalities are obtained:

$$
\begin{aligned}
\dot{V} & \leq k\left\|\Psi_{\varepsilon_{b d}}\right\|\left\|\omega_{b l}^{b}\right\|-\lambda_{\min }(L)\left\|\omega_{b l}^{b}\right\|^{2} \\
& \leq k \kappa_{3}\left\|\omega_{b l}^{b}\right\|-\lambda_{\min }(L)\left\|\omega_{b l}^{b}\right\|^{2} \\
& \leq k \kappa_{3}\left\|\begin{array}{c}
\varepsilon_{b d} \| \\
\omega_{b l}^{b}
\end{array}\right\|-\lambda_{\min }(L)\left\|\begin{array}{c}
\varepsilon_{b d} \\
\omega_{b l}^{b}
\end{array}\right\|^{2}+\lambda_{\min }(L)\left\|\varepsilon_{b d}\right\|^{2} \\
& \leq k \kappa_{3}\left\|\begin{array}{c}
\varepsilon_{b d} \\
\omega_{b l}^{b}
\end{array}\right\|-\lambda_{\min }(L)\left\|\begin{array}{c}
\varepsilon_{b d} \\
\omega_{b l}^{b}
\end{array}\right\|^{2}+\lambda_{\min }(L) \\
& =\dot{V}_{1},
\end{aligned}
$$

TABLE 1: Parameter simulation for single spacecraft case.

\begin{tabular}{lc}
\hline Parameters & \multicolumn{3}{c}{ Value } \\
\hline$q_{d l}[22]$ & {$\left[\begin{array}{ccc}1.49 & 0.054 & 0.0442 \\
0.054 & 1.51 & 0 \\
0.0442 & 0 & 1.56\end{array}\right]$} \\
$q_{b l}(0)$ & {$\left[\begin{array}{cccc}1 & 0 & 0 & 0\end{array}\right]^{T}$} \\
$\omega_{b l}^{b}(0) ; \omega_{d l}^{b}(t), t \geq 0$ & {$\left[\begin{array}{cccc}1 / 3 & 0 & \sqrt{1 / 3} & \sqrt{1 / 3}\end{array}\right]^{T}$} \\
$k$ & {$\left[\begin{array}{lll}0 & 0 & 0\end{array}\right]^{T}$} \\
$L$ & {$\left[\begin{array}{ccc}1 & 0 & 0 \\
0 & 1 & 0 \\
0 & 0 & 1\end{array}\right]$} \\
$\bar{\phi}$ & 0.57 \\
\hline
\end{tabular}

where $\left\|\Psi_{\varepsilon_{b d}}\right\| \leq \kappa_{3} \leq \kappa_{1}$ and $\dot{V}_{1}$ is given as follows:

$$
\begin{aligned}
\dot{V}_{1}= & -(1-\theta) \lambda_{\min }(L)\|\| \begin{array}{c}
\varepsilon_{b d} \\
b
\end{array}\left\|_{b l}^{2}-\theta \lambda_{\min }(L)\right\| \begin{array}{c}
\varepsilon_{b d} \\
b
\end{array} \|^{b} \\
& +k \kappa_{3}\left\|\begin{array}{c}
\varepsilon_{b d} \\
\omega_{b l}^{b}
\end{array}\right\|+\lambda_{\min }(L), \quad 0<\theta<1 .
\end{aligned}
$$

Therefore, inequality (34) is fulfilled for every $\left\|\begin{array}{c}\varepsilon_{b d} \\ \omega_{b l}^{b}\end{array}\right\|$ satisfying (35):

$$
\begin{gathered}
\dot{V} \leq-(1-\theta) \lambda_{\min }(L)\left\|\begin{array}{c}
\varepsilon_{b d} \\
\omega_{b l}^{b}
\end{array}\right\|^{2}, \\
\left\|\begin{array}{c}
\varepsilon_{b d} \\
\omega_{b l}^{b}
\end{array}\right\| \geq \frac{k \kappa_{3}+\sqrt{\left(k \kappa_{3}\right)^{2}+4 \theta \lambda_{\text {min }}{ }^{2}(L)}}{2 \theta \lambda_{\min }(L)}=\rho .
\end{gathered}
$$

In according to Theorem 4.18 in [20], solution of the system is ultimately bounded with the ultimate bound as follows:

$$
\left\|\varepsilon_{b d}\right\| \leq \alpha_{1}^{-1}\left(\alpha_{2}(\rho)\right)
$$

Remark 4. Note that $\kappa_{3}$ in (35) clearly satisfies $\left\|\Psi_{\varepsilon_{b d}}\right\| \leq \kappa_{3} \leq$ $\kappa_{1}$. This fact implies that $\rho$ in (35) is possible to be smaller when $\varepsilon_{b d}$ is getting smaller. Since $\rho$ is getting smaller, then the ultimate bound is smaller. Thus, if $\lim _{t \rightarrow \infty} \rho=0$, then it will be equivalent to asymptotic stability of the set consisting

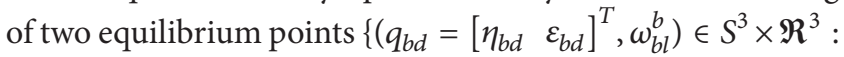
$\left.\varepsilon_{b d}=0, \omega_{b l}^{b}=0\right\}$.

The solid line in Figure 1 depicts the response of Euler angle attitude control system designed based on Theorem 3 using parameters in Table 1 . The response is also compared by Euler Angle response of the attitude control system (4)-(6) 


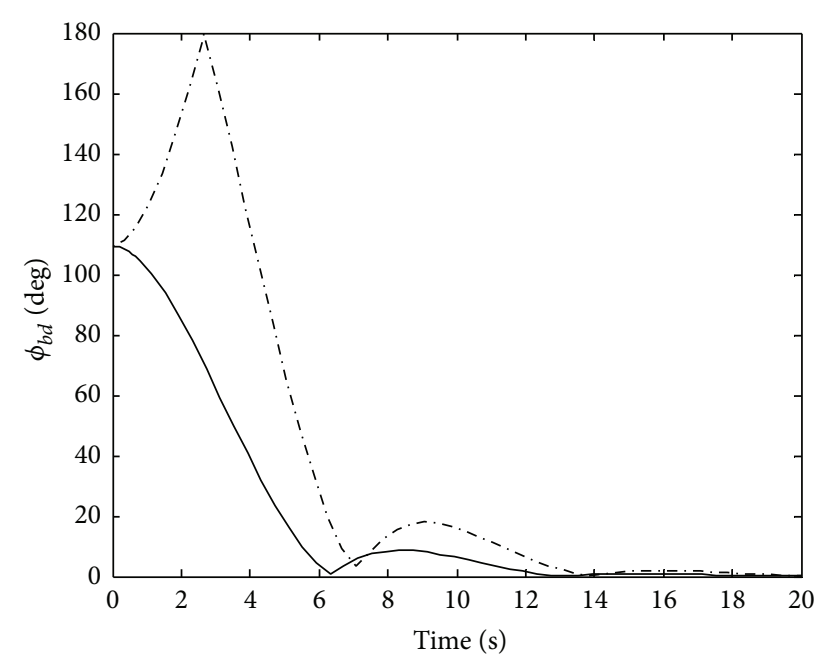

FIgURE 1: Euler angle response, $\phi_{b d}$; Theorem 3 (solid line) and PDlike control law (dash-dot line).

using a well-known PD-like control law, $\tau=-k \varepsilon_{b d}-L \omega_{b d}^{b}-$ depicted by dash-dot line.

As seen from Figure 1, the attitude control system employing PD-like control law exhibits unwinding phenomenon because it is designed by considering only one equilibrium point in quaternion parameterization. Figure 2 shows more clearly that unlike the attitude control system employing PDlike control law, the designed control system converges to the closer equilibrium point, $\left[\begin{array}{llll}-1 & 0 & 0 & 0\end{array}\right]$. Figure 3 shows the opposite direction of rotation between the two control systems. This situation implies the efficiency of energy consumption as shown in Figure 4. In addition, the responses depicted in Figures 2 and 3 show that the situation explained in Remark 4 is promising.

Now, suppose that the external disturbance of system (6) is continuous and bounded, that is,

$$
\|d(t)\| \leq c, \quad d \in \mathfrak{R}^{3}, c \in \mathfrak{R}, t \geq 0 .
$$

Theorem 5. If scalar $k$ is positive and matrix $L$ is symmetric and positive definite, quaternion-based attitude control system composed by (4)-(6) and (12) is input to state stable.

Proof. Using the same energy-like function (23), its time derivative is given as follows:

$$
\dot{V}=k \omega_{b l}^{b^{T}} \Psi_{\varepsilon_{b d}}-\omega_{b l}^{b^{T}} L \omega_{b l}^{b}+\omega_{b l}^{b^{T}} d .
$$

Note that, according to (37), the disturbance is bounded by $\|d\| \leq c$. Following the way of proof of Theorem 3 , for $0<\theta<$ $1,(40)$ is satisfied:

$$
\dot{V} \leq-(1-\theta) \lambda_{\min }(L)\left\|\begin{array}{c}
\varepsilon_{b d} \\
\omega_{b l}^{b}
\end{array}\right\|
$$

where $\kappa_{3}$ satisfies $\left\|\Psi_{\varepsilon_{b d}}\right\| \leq \kappa_{3} \leq \kappa_{1}, \kappa_{1}$ is the same as (29) and every $\left\|_{\omega_{b l}^{b}}^{\varepsilon_{b}}\right\|$ satisfies $(40)$ :

$$
\left\|\begin{array}{c}
\varepsilon_{b d} \\
\omega_{b l}^{b}
\end{array}\right\| \geq \frac{\left(k \kappa_{3}+c\right)+\sqrt{\left(k \kappa_{3}+c\right)^{2}+4 \theta \lambda_{\min }^{2}(L)}}{2 \theta \lambda_{\min }(L)}=\rho_{\text {ISS }}(c) .
$$

According to Theorem 4.19 in [20], the system is input to state stable with ultimate bound of the system's solution being as follows:

$$
\left\|\begin{array}{c}
\varepsilon_{b d} \\
\omega_{b l}^{b}
\end{array}\right\| \leq \alpha_{1}^{-1}\left(\alpha_{2}\left(\rho_{\text {ISS }}(c)\right)\right) .
$$

To verify Theorem 5, a simulation is run using the same parameters in Table 1 and a disturbance function,

$$
d(t)=\left[\begin{array}{c}
3 \cdot 10^{-2} \sin (10 \pi t) \\
5 \cdot 10^{-2} \sin (10 \pi t) \\
2 \cdot 10^{-2} \sin \left(10 \pi t-\frac{\pi}{2}\right)
\end{array}\right]
$$

as shown in Figure 5. The boundedness of solution properties of the designed attitude control system in the presence of disturbance is confirmed by Figures 6 and 7. Figure 9 shows the control action of the designed control system in order to have robustness property in the presence of disturbance as shown in inset of Figure 6. In addition, Figure 8 is presented as comparison control action without the presence of disturbance.

3.3. Cooperative Spacecraft Case. Bounded solution of cooperative spacecraft attitude control system involves attitude errors between spacecrafts, that is, $\left\|\begin{array}{c}\varepsilon_{b(i) b(j)} \\ \omega_{b(i) l}^{b(i)}\end{array}\right\|$. In this subsection it will be shown that through the boundedness of solution approach, cooperative attitude system could be designed.

Theorem 6. Consider the cooperative spacecraft attitude control system composed by (7)-(10) and (13). Suppose scalar $k$ is positive and matrix $L$ and moment of inertia $J_{(i)}$ are symmetric and positive definite, for $1 \leq i \leq n \in Z_{>0}$. Suppose external disturbance $_{(i)}$ is continuous and bounded function, $\left\|d_{(i)}\right\| \leq c$, $c \in \mathfrak{R}$, for $1 \leq i \leq n \in Z_{>0}$. If at least there is a directed spanning tree in the topology representing information-state exchange between spacecraft $i$ and spacecraft $j$ in a group composed by n-spacecrafts, then solution of the cooperative system is bounded.

Proof. Consider the energy-like function (43):

$$
V=k \sum_{i, j}^{n} a_{(i)(j)} \varepsilon_{b(i) b(j)}^{T} \varepsilon_{b(i) b(j)}+\sum_{i}^{n} \frac{1}{2} \omega_{b(i) l}^{b(i)}{ }^{T} J_{(i)} \omega_{b(i) l}^{b(i)} .
$$




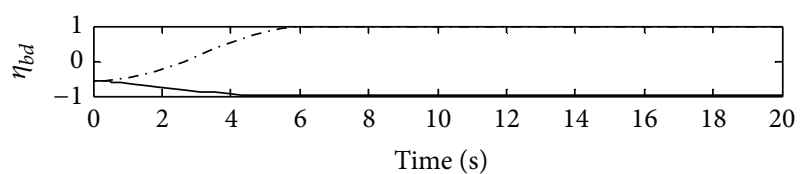

(a)

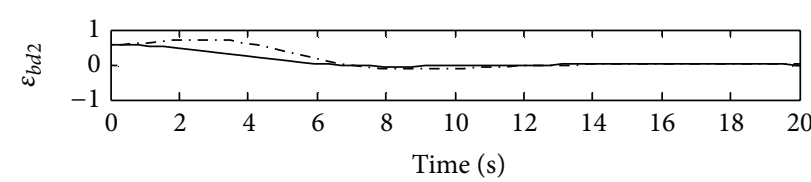

(c)

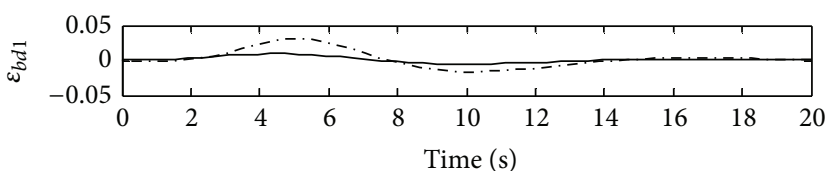

(b)

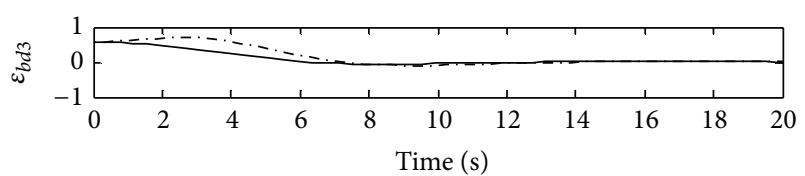

(d)

FIGURE 2: Attitude responses, $q_{b d}=\left[\begin{array}{ll}\eta_{b d} & \varepsilon_{b d}\end{array}\right]^{T}=\left[\begin{array}{llll}\eta_{b d} & \varepsilon_{b d 1} & \varepsilon_{b d 2} & \varepsilon_{b d 3}\end{array}\right]^{T}$; Theorem 3 (solid line) and PD-like control law (dash-dot line).

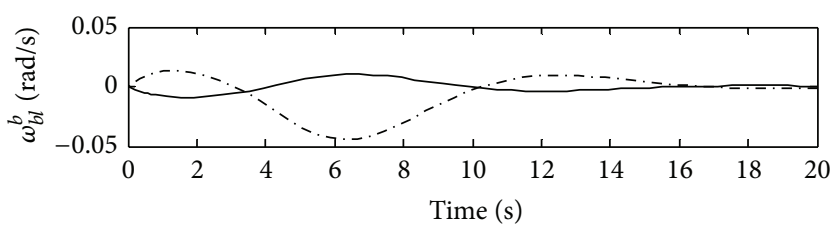

(a)

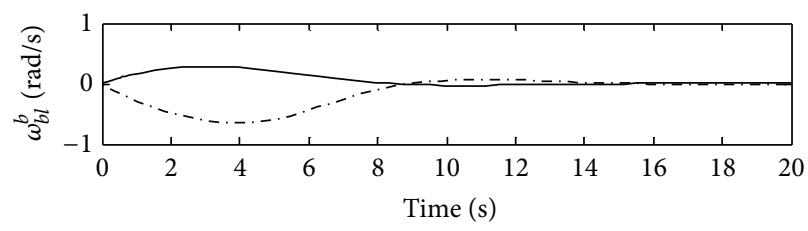

(b)

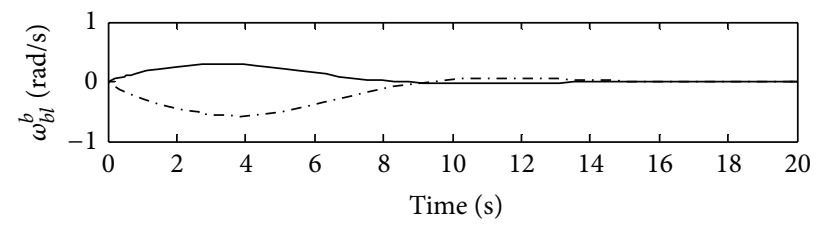

(c)

Figure 3: Angular velocity; Theorem 3 (solid line) and PD-like control law (dash-dot line).

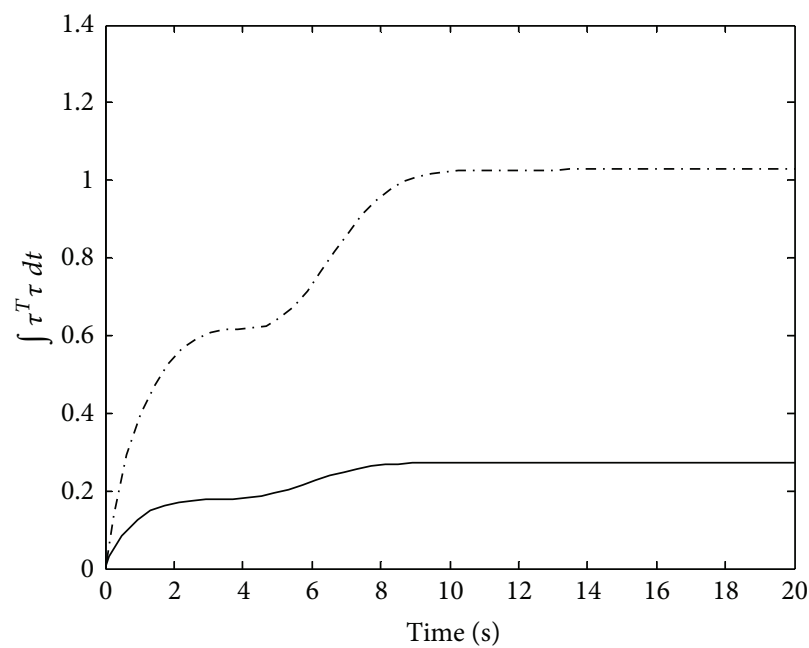

FIgURE 4: Energy consumption; Theorem 3 (solid line) and PD-like control law (dash-dot line). 


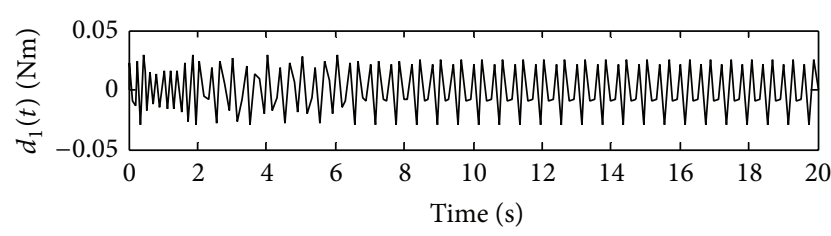

(a)

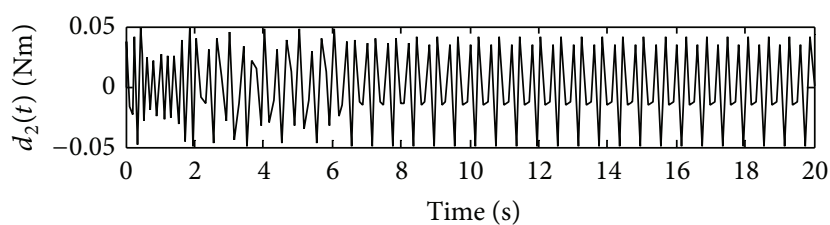

(b)

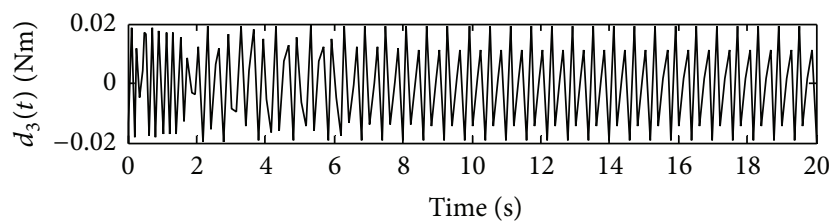

(c)

FIGURE 5: External disturbance; $d(t)=\left[\begin{array}{lll}d_{1}(t) & d_{2}(t) & d_{3}(t)\end{array}\right]^{T}$.

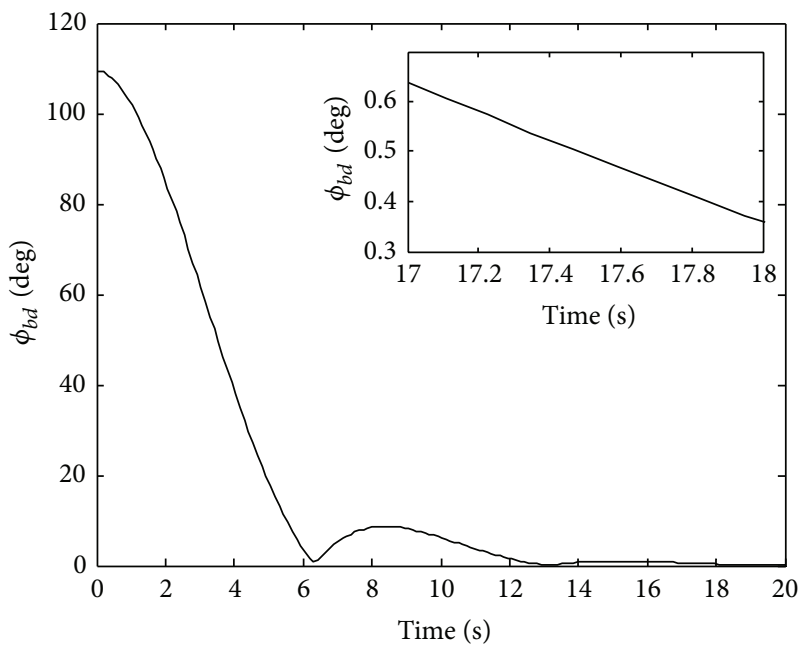

Figure 6: Euler angle response, $\phi_{b d}$; Theorem 5.

Then, its time derivatives can be written as follows:

$$
\begin{aligned}
\dot{V}= & k \sum_{i, j}^{n} a_{(i)(j)} \varepsilon_{b(i) b(j)}^{T} \eta_{b(i) b(j)} \omega_{b(i) b(j)}^{b(i)} \\
& +\sum_{i}^{n} \omega_{b(i) l}^{b(i)^{T}} k \sum_{j}^{n} a_{(i)(j)}\left(-\eta_{b(i) b(j)} \varepsilon_{b(i) b(j)}+\Psi_{\varepsilon_{b(i) b(j)}}-L \omega_{b(i) l}^{b(i)}\right) \\
& +\sum_{i}^{n} \omega_{b(i) l}^{b(i)} d_{(i)} .
\end{aligned}
$$

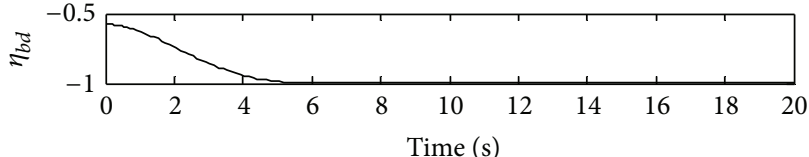

(a)

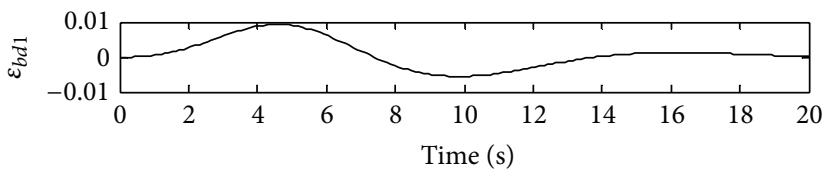

(b)

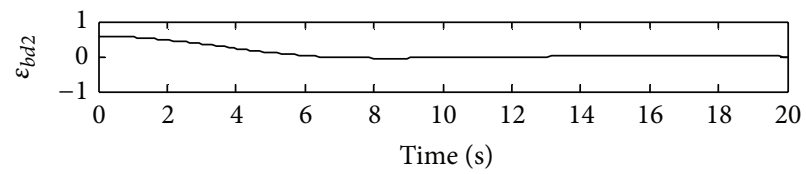

(c)

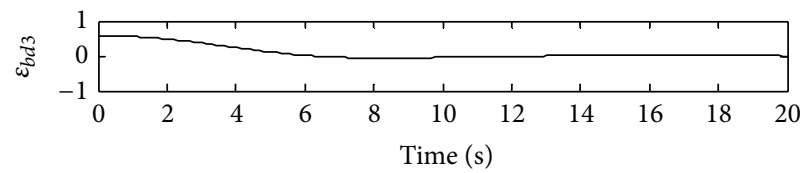

(d)

Figure 7: Attitude responses; Theorem 5.

Since $\omega_{b(i) b(j)}^{b(i)}=\omega_{b(i) l}^{b(i)}-R_{b(i) b(j)} \omega_{b(j) l}^{b(j)}=\omega_{b(i) l}^{b(i)}-\omega_{b(j) l}^{b(i)}$, then $(45)$ is satisfied.

$$
\begin{aligned}
\dot{V}= & -k \sum_{i, j}^{n} a_{(i)(j)} \varepsilon_{b(i) b(j)}^{T} \eta_{b(i) b(j)} \omega_{b(j) l}^{b(i)} \\
& +\sum_{i}^{n} \omega_{b(i) l}^{b(i)}{ }^{T} k \sum_{j}^{n} a_{(i)(j)}\left(\Psi_{\varepsilon_{b(i) b(j)}}-L \omega_{b(i) l}^{b(i)}\right) \\
& +\sum_{i}^{n} \omega_{b(i) l}^{b(i)}{ }^{T} d_{(i)} .
\end{aligned}
$$

Regarding property of spacecraft orientation and angular velocity, there is inequality as follows:

$$
\begin{aligned}
\dot{V} \leq & k \sum_{i, j}^{n} a_{(i)(j)}\left\|\eta_{b(i) b(j)} \varepsilon_{b(i) b(j)}\right\| \cdot\left\|\omega_{b(j) l}^{b(i)}\right\| \\
& +k \sum_{i, j}^{n} a_{(i)(j)} \omega_{b(i) l}^{b(i)}{ }^{T}\left(\Psi_{\varepsilon_{b(i) b(j)}}-L \omega_{b(i) l}^{b(i)}\right)+\sum_{i}^{n} \omega_{b(i) l}^{b(i) T} d_{(i)} .
\end{aligned}
$$

Noting that $\left\|\eta_{b(i) b(j)} \varepsilon_{b(i) b(j)}\right\| \leq 1 / 2,\left\|d_{(i)}\right\| \leq c$ and the bound of $\left\|\Psi_{\varepsilon_{b(i) b(j)}}\right\|$ then the following inequality can be obtained:

$$
\begin{aligned}
\dot{V} \leq & \frac{1}{2} \sum_{i, j}^{n} a_{(i)(j)}\left\|\omega_{b(j) l}^{b(i)}\right\|+k \kappa_{3} \sum_{i, j}^{n} a_{(i)(j)}\left\|\omega_{b(i) l}^{b(i)}\right\| \\
& -k \sum_{i, j}^{n} a_{(i)(j)} \lambda_{\min }(L)\left\|\omega_{b(i) l}^{b(i)}\right\|^{2}+c \sum_{i}^{n}\left\|\omega_{b(i) l}^{b(i)}\right\|,
\end{aligned}
$$




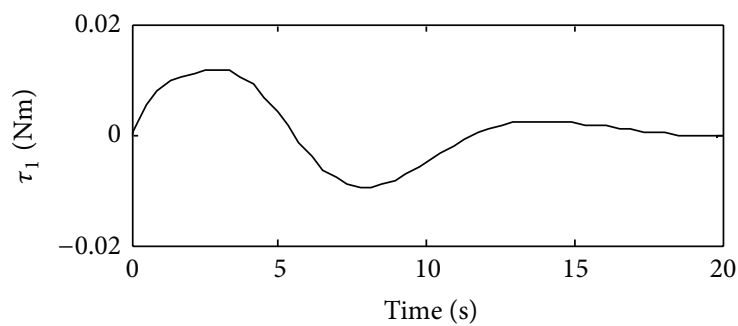

(a)

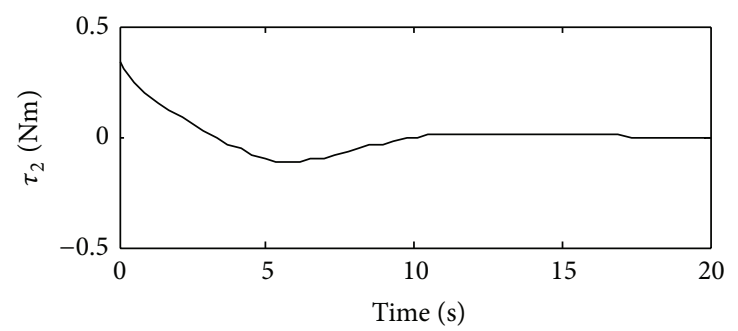

(c)

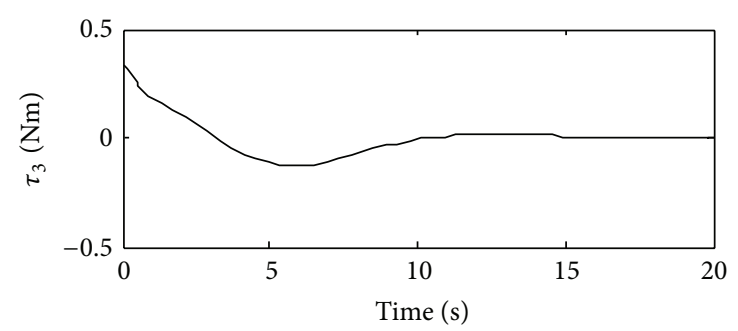

(e)

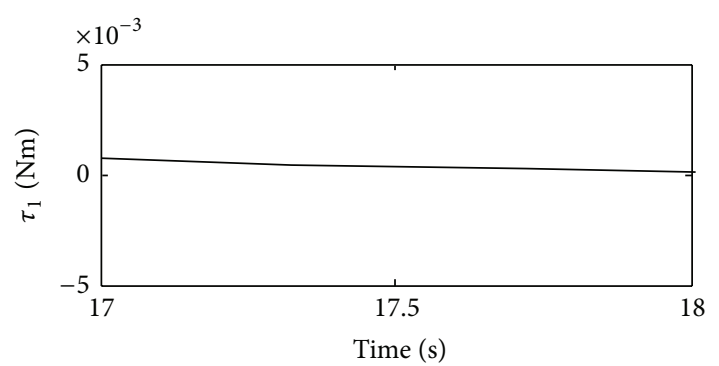

(b)

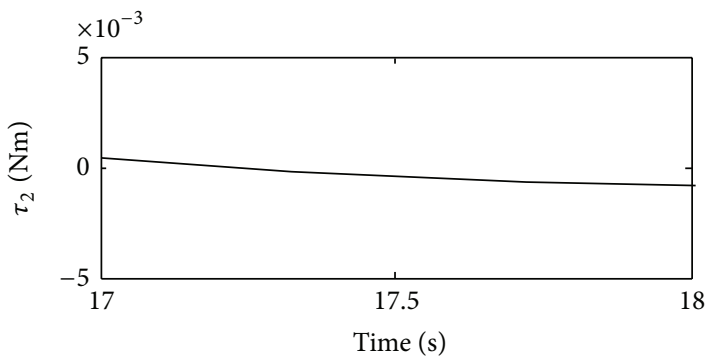

(d)

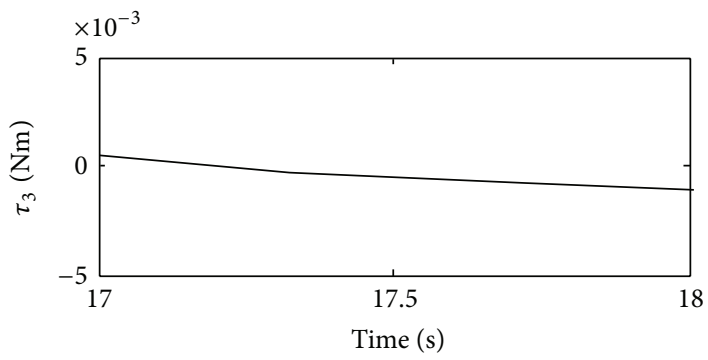

(f)

Figure 8: Control torque, $\tau=\left[\begin{array}{lll}\tau_{1} & \tau_{2} & \tau_{3}\end{array}\right]^{T}$; Theorem 3 .

where $\left\|\Psi_{\varepsilon_{b(i) b(j)}}\right\| \leq \kappa_{3} \leq \kappa_{1}=\max \left\{3 \cdot(\sqrt{1 / 3}-\alpha)^{2},(1-\alpha)^{2}\right\}<$ 1. And

$$
\begin{aligned}
\dot{V} \leq \dot{\bar{V}}= & k \frac{1}{2} \sum_{i, j}^{n} a_{(i)(j)}\left\|\begin{array}{c}
\omega_{b(j) l}^{b(i)} \\
\varepsilon_{b(i) b(j)}
\end{array}\right\|+k \kappa_{3} \sum_{i, j}^{n} a_{(i)(j)}\left\|\begin{array}{c}
\omega_{b(i) l}^{b(i)} \| \varepsilon_{b(i) b(j)}
\end{array}\right\| \\
& -k \sum_{i, j}^{n} a_{(i)(j)} \lambda_{\min }(L)\left\|\begin{array}{c}
\omega_{b(i) l}^{b(i)} \\
\varepsilon_{b(i) b(j)}
\end{array}\right\|^{2} \\
& +k \sum_{i, j}^{n} a_{(i)(j)} \lambda_{\min }(L)\left\|\varepsilon_{b(i) b(j)}\right\|^{2}+c \sum_{i}^{n}\left\|\omega_{b(i) l}^{b(i)}\right\| .
\end{aligned}
$$

Therefore, there are $k>0$ and symmetric matrix $L>0$ such that $\dot{\bar{V}}$ implies that $\left\|\begin{array}{c}\omega_{b(i) l}^{b(i)} \\ \varepsilon_{b(i) b(j)}\end{array}\right\|$ is bounded for $1 \leq i, j \leq n$ and $t \geq 0$.
Now, suppose $\left\|\begin{array}{c}\omega_{b(i) l}^{b(i)} \\ \varepsilon_{b(i) b(j)}\end{array}\right\|$ is not bounded. Without loss of generality, taking $k=\lambda_{\min }(L)=c=1$, the following inequality is obtained:

$$
\begin{aligned}
\dot{\bar{V}} \leq \dot{\widehat{V}}= & \frac{1}{2} \sum_{i, j}^{n} a_{(i)(j)}\left\|\begin{array}{c}
\omega_{b(j) l}^{b(i)} \\
\varepsilon_{b(i) b(j)}
\end{array}\right\|+\sum_{i, j}^{n} a_{(i)(j)}\left\|\begin{array}{c}
\omega_{b(i) l}^{b(i)} \| \\
\varepsilon_{b(i) b(j)}
\end{array}\right\| \\
& -\sum_{i, j}^{n} a_{(i)(j)}\left\|\begin{array}{c}
\omega_{b(i) l}^{b(i)} \\
\varepsilon_{b(i) b(j)}
\end{array}\right\|^{2}+\sum_{i, j}^{n} a_{(i)(j)}\left\|\varepsilon_{b(i) b(j)}\right\|^{2} \\
& +\sum_{i}^{n}\left\|\omega_{b(i) l}^{b(i)}\right\| .
\end{aligned}
$$

Then, there is a sufficiently large $\left\|\begin{array}{c}\omega_{b(i) l}^{b(i)} \\ \varepsilon_{b(i) b(j)}\end{array}\right\|$, where, inherently, also sufficiently large $\left\|\begin{array}{c}\omega_{b(j) l}^{b(i)} \\ \varepsilon_{b(i) b(j)}\end{array}\right\|=\left\|\begin{array}{c}R_{b(i) b(j)} \omega_{b(j) l}^{b(j)} \\ \varepsilon_{b(i) b(j)}\end{array}\right\|$ and $\left\|\omega_{b(i) l}^{b(i)}\right\|$, such that $\dot{\widehat{V}}>0$, and the increasing $\left\|\begin{array}{c}\omega_{b(i) l}^{b(i)} \\ \varepsilon_{b(i) b(j)}\end{array}\right\|$ are resulting $\dot{\hat{V}}>0$. 


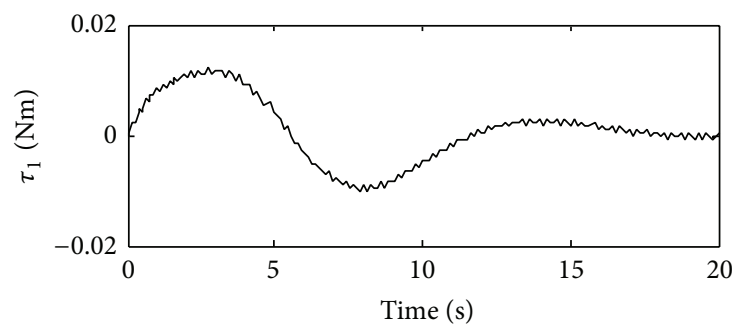

(a)

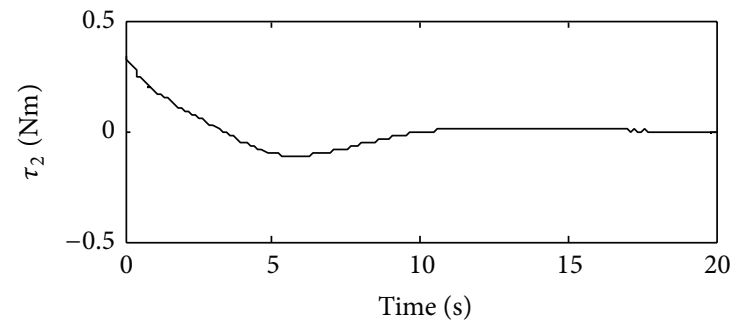

(c)

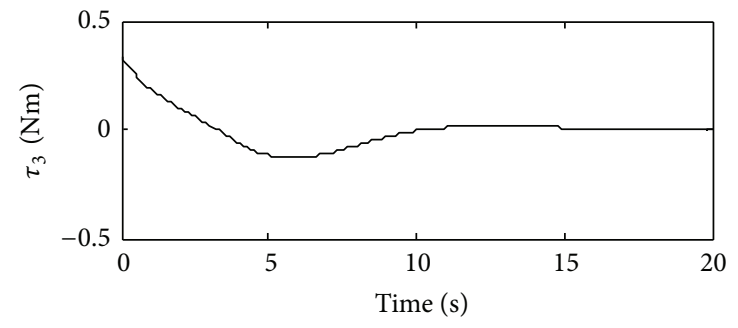

(e)

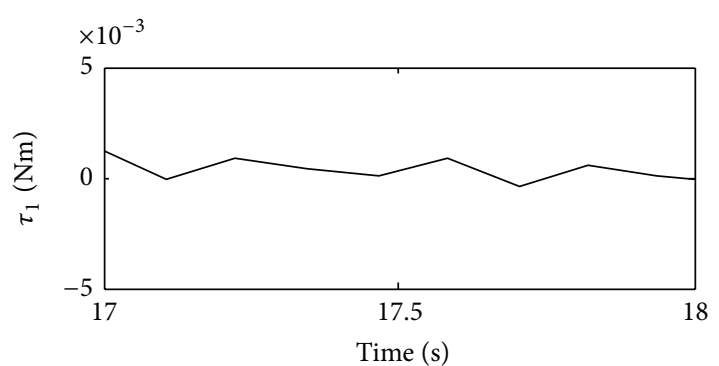

(b)

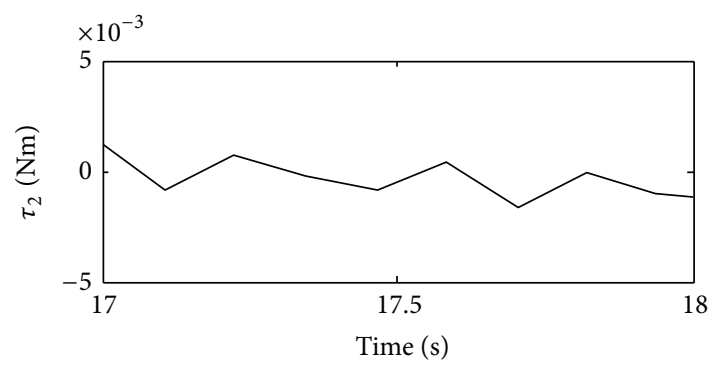

(d)

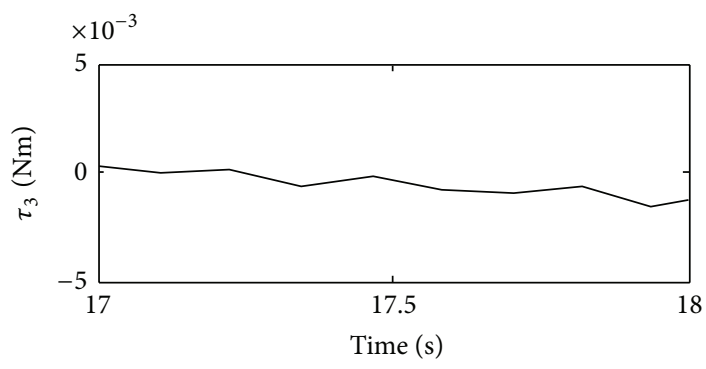

(f)

Figure 9: Control torque; Theorem 5.

Let the information-state exchange topology consist of one directed spanning tree and $\left\|\begin{array}{c}\omega_{b(i) l}^{b(i)} \\ \varepsilon_{b(i)(j)} \|\end{array}\right\|=\sigma \neq \infty$; therefore, the following inequality is obtained:

$$
\begin{aligned}
\dot{\widehat{V}}<\dot{\widetilde{V}} & =\frac{1}{2}(n-1) \sigma+(n-1) \sigma-(n-1) \sigma^{2}+(n-1)+n \sigma \\
& =-(n-1) \sigma^{2}+\frac{1}{2}(5 n-3) \sigma+(n-1) .
\end{aligned}
$$

This contradicts that, for $\sigma>((1 / 2)(5 n-3)+$ $\sqrt{\left.((1 / 2)(5 n-3))^{2}+4(n-1)\right)} / 2(n-1), \dot{\bar{V}}<0$. Note that it implies that $\dot{V}<0$. Therefore, by contradiction, it is shown that $\left\|\omega_{\varepsilon_{b(i) b(j) l}(j)}^{b_{(i)}}\right\|$ is bounded.

Remark 7. Through the proof by contradiction described above, solution of the cooperative system $\left\|\varepsilon_{\varepsilon_{b(i) b(j)}}^{\omega_{b(i) l}^{(i)}}\right\|$ is bounded for all $k>0$ and symmetric matrix $L>0$ where that only requires a directed spanning tree in information-state exchange topology.

Remark 8 . Note that here the condition of informationstate exchange topology is less-conservative condition, for example, directed spanning tree. In contrast, for example, [15] requires acyclic topology and [16] requires connected (undirected) topology.

Simulation of cooperative attitude control case is run using $\bar{\phi}, k$, and $L$ as shown in Table 1 and the same disturbance function as used in the previous subsection. The simulation used three spacecrafts with different moment of inertia and three different initial attitudes as shown in Table 2. Meanwhile, initial angular velocities of all spacecrafts are zero. A cyclic directed graph representing information-state exchange is applied in the simulation (Figure 10). Figure 11 shows that each spacecraft converges to absolute attitude about $95^{\circ}$. It implies, as shown in Figure 12, that the relative attitudes between spacecrafts are regulated. 


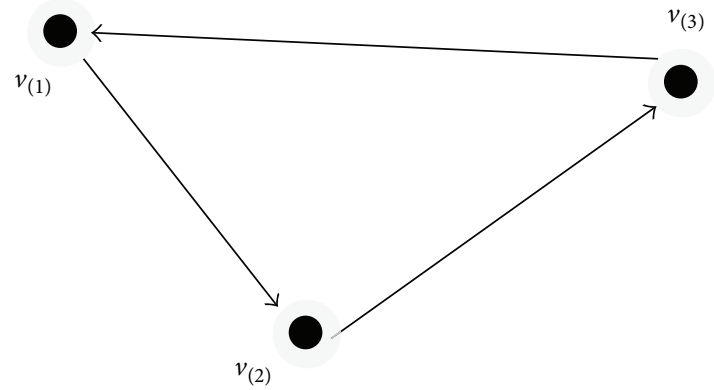

FIGURE 10: Information-state exchange topology.

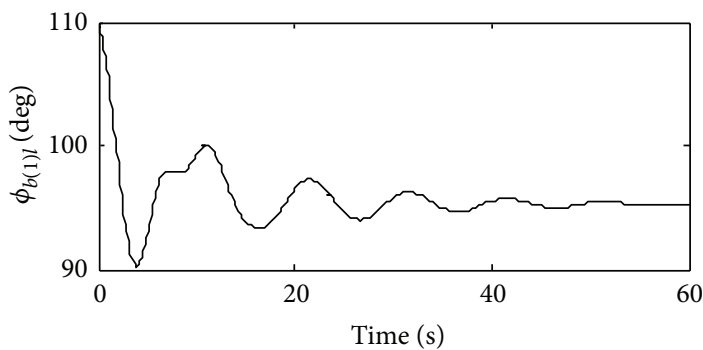

(a)

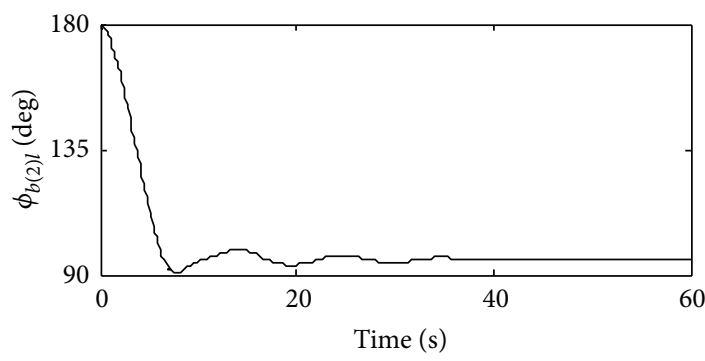

(c)

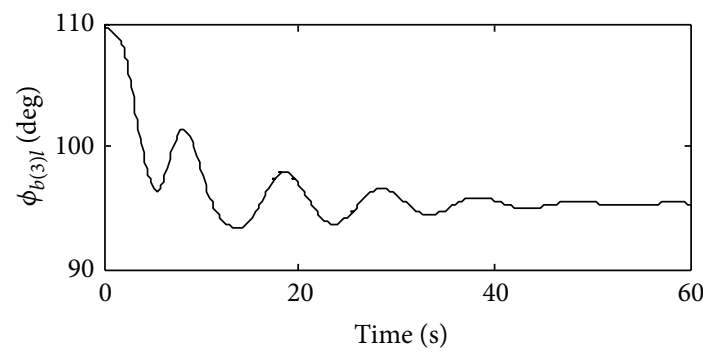

(e)

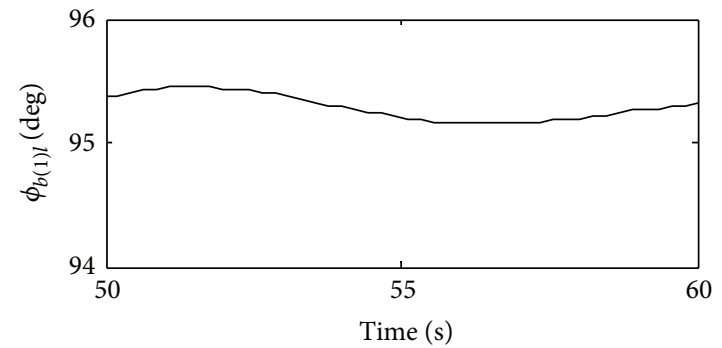

(b)

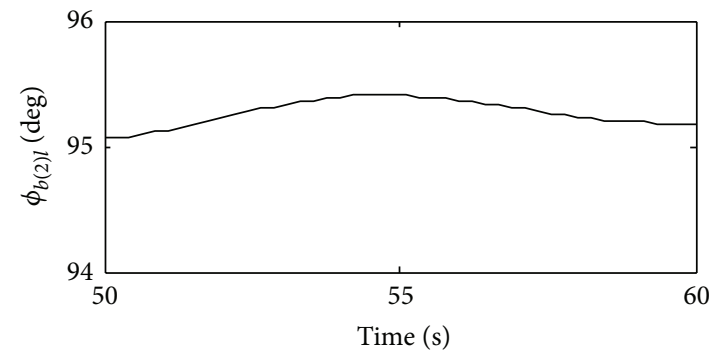

(d)

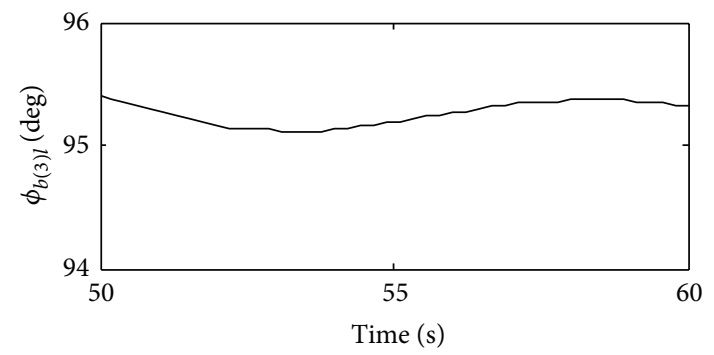

(f)

FIgure 11: Absolute Euler angles; Theorem 6.

\section{Concluding Remarks}

Dynamics of spacecraft rotational motion based on Euler equation and quaternion-based kinematics describing the spacecraft attitudes have been presented. For cooperative spacecrafts case, information-state exchange is modeled by directed graph. Using these models, design of attitude control through boundedness of solution approach for single and cooperative spacecrafts have been proposed. The control designs are done by regarding two equilibrium points.

In single spacecraft attitude control system case, the attitude control system has been designed, for zero disturbances, via ultimately bounded solution and for nonzero disturbances, via input to state stability approach. For zero disturbances, if the ultimate bound is zero at $t \rightarrow \infty$, then it is equivalent to asymptotic stability. In cooperative spacecraft 


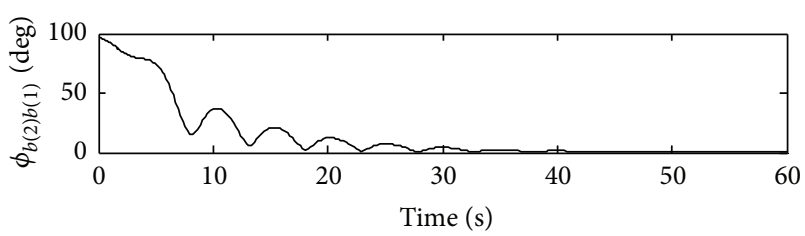

(a)

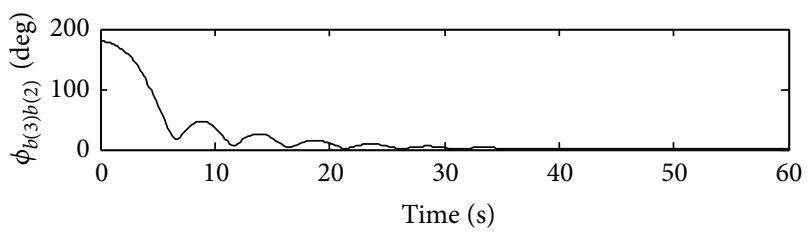

(b)

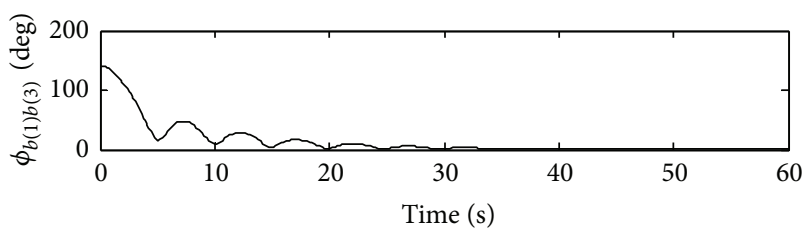

(c)

FIGURE 12: Relative Euler angle between spacecrafts; Theorem 6.

TABLE 2: Parameter simulation for cooperative spacecrafts case.

\begin{tabular}{|c|c|c|c|}
\hline Parameters & & Value & \\
\hline & 1.49 & 0.054 & 0.442 \\
\hline$J_{(1)}$ & 0.054 & 1.51 & 0 \\
\hline & 0.0442 & 0 & 1.56 \\
\hline & 1.49 & 0 & 0.04427 \\
\hline$J_{(2)}$ & 0 & 1.51 & 0.054 \\
\hline & 0.0442 & 0.054 & 1.56 \\
\hline & {$[1.49$} & 0 & $0.054]$ \\
\hline$J_{(3)}$ & 0 & 1.56 & 0.442 \\
\hline & 0.054 & 0.442 & 1.51 \\
\hline$q_{(1) l}(0)$ & {$\left[\begin{array}{ll}-\sqrt{1 / 3} & 0\end{array}\right.$} & $0 \quad \sqrt{1 / 3}$ & $\overline{3} \quad \sqrt{1 / 3}]^{T}$ \\
\hline$q_{(2) l}(0)$ & {$\left[\begin{array}{ll}0 & \sqrt{1 / 3}\end{array}\right.$} & $\sqrt{1 / 3}$ & $\sqrt{1 / 3}]^{T}$ \\
\hline$q_{(3) l}(0)$ & {$\left[\begin{array}{ll}\sqrt{1 / 3} & 0\end{array}\right.$} & $\sqrt{1 / 3}$ & $-\sqrt{1 / 3}]^{T}$ \\
\hline
\end{tabular}

attitude control system case, the cooperative system has been designed via the boundedness of solution approach. If the information-state exchange topology has directed spanning tree, then the cooperative spacecraft attitude system is bounded. In addition, all theorems stated in this paper have been verified in simulations. The transient responses and steady state responses demonstrate effectiveness of the proposed methodology.

\section{Conflict of Interests}

The authors declare that there is no conflict of interests regarding the publication of this paper.

\section{Acknowledgments}

This research is partly supported by Beasiswa Unggulan, BPKLN, Kemendikbud, Indonesia. The authors also gratefully acknowledge the referees for carefully reading the paper and many helpful comments.

\section{References}

[1] E. R. Pinheiro and L. C. G. de Souza, "Design of the microsatellite attitude control system using the mixed $\mathrm{H}_{2} / \mathrm{H}_{\infty}$ method via LMI optimization," Mathematical Problems in Engineering, vol. 2013, Article ID 257193, 8 pages, 2013.

[2] L.-L. Show, J.-C. Juang, and Y.-W. Jan, "An LMI-based nonlinear attitude control approach," IEEE Transactions on Control Systems Technology, vol. 11, no. 1, pp. 73-83, 2003.

[3] R. Schlanbusch, A. Loria, R. Kristiansen, and P. J. Nicklasson, "PD+ based output feedback attitude control of rigid bodies," IEEE Transactions on Automatic Control, vol. 57, no. 8, pp. 21462152, 2012.

[4] H. Septanto, B. Riyanto-Trilaksono, A. Syaichu-Rohman, and R. Eko Poetro, "On the stability analysis of a discontinuous quaternion attitude control system," in Proceedings of the International Conference on System Engineering and Technology (ICSET '12), pp. 1-4, Bandung, Indonesia, September 2012.

[5] C. G. Mayhew, R. G. Sanfelice, and A. R. Teel, "Quaternionbased hybrid control for robust global attitude tracking," IEEE Transactions on Automatic Control, vol. 56, no. 11, pp. 25552566, 2011.

[6] J. Stuelpnagel, "On the parameterization of the threedimensional rotation group," SIAM Review, vol. 6, no. 4, pp. 422-430, 1964.

[7] S. P. Bhat and D. S. Bernstein, "A topological obstruction to continuous global stabilization of rotational motion and the unwinding phenomenon," Systems and Control Letters, vol. 39, no. 1, pp. 63-70, 2000.

[8] S. Li, S. Ding, and Q. Li, "Global set stabilization of the spacecraft attitude control problem based on quaternion," International Journal of Robust and Nonlinear Control, vol. 20, no. 1, pp. 84-105, 2010.

[9] R. Kristiansen, P. J. Nicklasson, and J. T. Gravdahl, "Satellite attitude control by quaternion-based backstepping," IEEE Transactions on Control Systems Technology, vol. 17, no. 1, pp. 227-232, 2009.

[10] C. G. Mayhew, R. G. Sanfelice, and A. R. Teel, “On path-lifting mechanisms and unwinding in quaternion-based attitude control," IEEE Transactions on Automatic Control, vol. 58, no. 5, pp. 1179-1191, 2013.

[11] H. Septanto, B. Riyanto-Trilaksono, A. Syaichu-Rohman, R. Eko Poetro, and A. R. Ibrahim, "The quaternion-based attitude control system with an augmented dynamic," in Proceedings of the 3rd International Conference on Instrumentation, Communications, Information Technology, and Biomedical Engineering (ICICI-BME '13), pp. 190-193, Bandung, Indonesia, November 2013. 
[12] G. Krieger, A. Moreira, H. Fiedler et al., "TanDEM-X: a satellite formation for high-resolution SAR interferometry," IEEE Transactions on Geoscience and Remote Sensing, vol. 45, no. 11, pp. 3317-3341, 2007.

[13] S. Persson, S. Veldman, and P. Bodin, "PRISMA-a formation flying project in implementation phase," Acta Astronautica, vol. 65, no. 9-10, pp. 1360-1374, 2009.

[14] W. Ren, "Distributed attitude alignment in spacecraft formation flying," International Journal of Adaptive Control and Signal Processing, vol. 21, no. 2-3, pp. 95-113, 2007.

[15] C. G. Mayhew, R. G. Sanfelice, J. Sheng, M. Arcak, and A. R. Teel, "Quaternion-based hybrid feedback for robust global attitude synchronization," IEEE Transactions on Automatic Control, vol. 57, no. 8, pp. 2122-2127, 2012.

[16] Q. Hu, Y. Lv, J. Zhang, and G. Ma, "Input-to-state stable cooperative attitude regulation of spacecraft formation flying," Journal of the Franklin Institute, vol. 350, no. 1, pp. 50-71, 2012.

[17] H. Septanto, B. Riyanto-Trilaksono, A. Syaichu-Rohman, and R. Eko Poetro, "The saturated quaternion control law with application for spacecraft formation flying," in Proceedings of the IEEE Conference on Control, Systems \& Industrial Informatics (ICCSII '12), pp. 59-63, Bandung, Indonesia, September 2012.

[18] H. Septanto, B. Riyanto-Trilaksono, A. Syaichu-Rohman, and R. Eko Poetro, "Analisis Solusi Sistem Kendali Sikap Satelit Berelemen Saturasi," in Seminar Nasional Teknik Elektro Unjani, Indonesian National Conference, 2013.

[19] P. C. Hughes, Spacecraft Attitude Dynamics, Dover, New York, NY, USA, 2004.

[20] H. K. Khalil, Nonlinear Systems, Prentice Hall, Upper Saddle River, NJ, USA, 3rd edition, 2002.

[21] D. S. Bernstein, Matrix Mathematics: Theory, Facts and Formulas, Princeton University Press, Princeton, NJ, USA, 2nd edition, 2009.

[22] D. S. Roldugin and P. Testani, "Spin-stabilized satellite magnetic attitude control scheme without initial detumbling," Acta Astronautica, vol. 94, no. 1, pp. 446-454, 2014. 

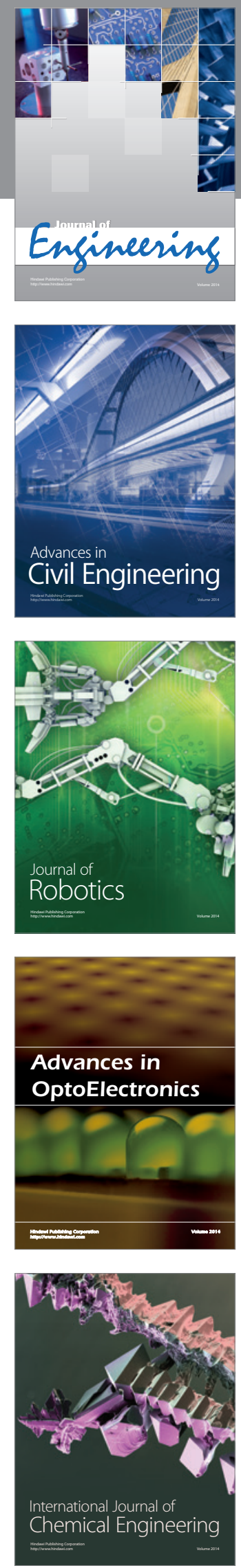

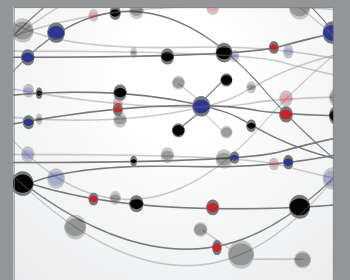

The Scientific World Journal
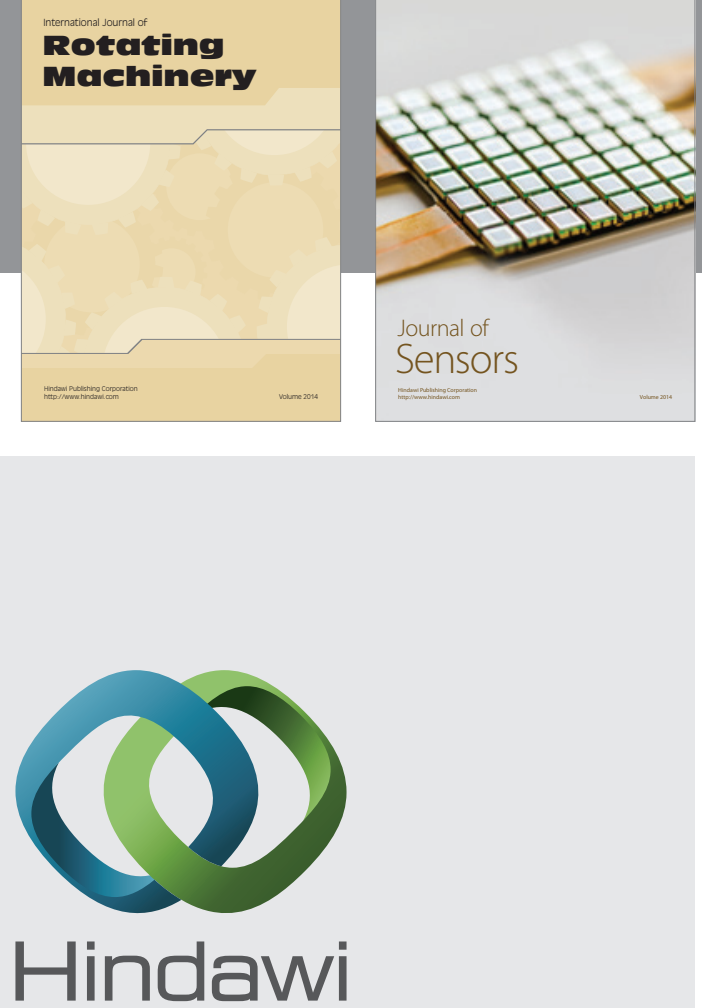

Submit your manuscripts at http://www.hindawi.com
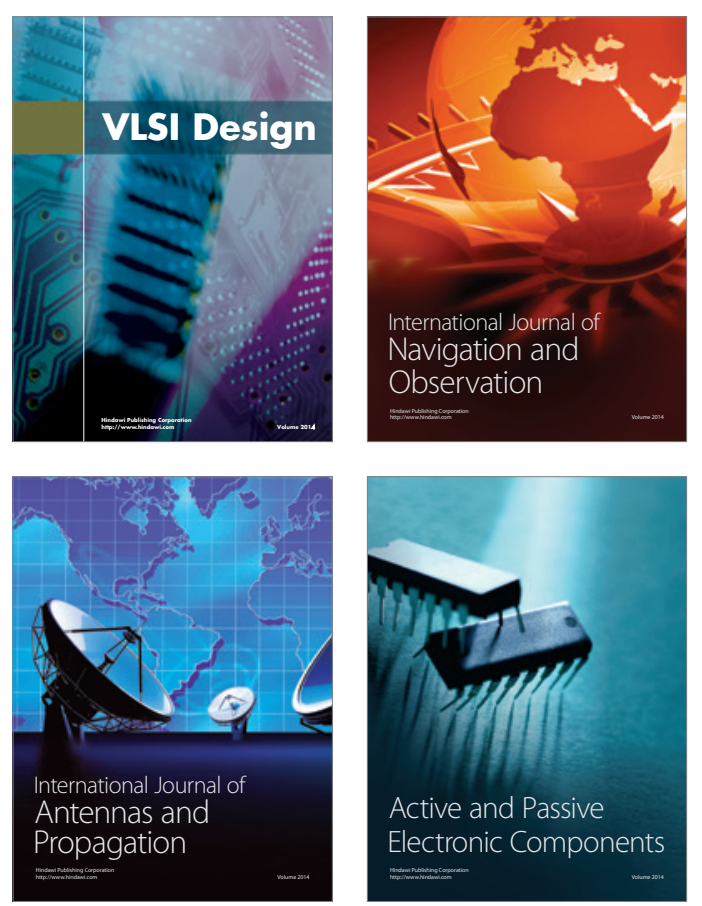
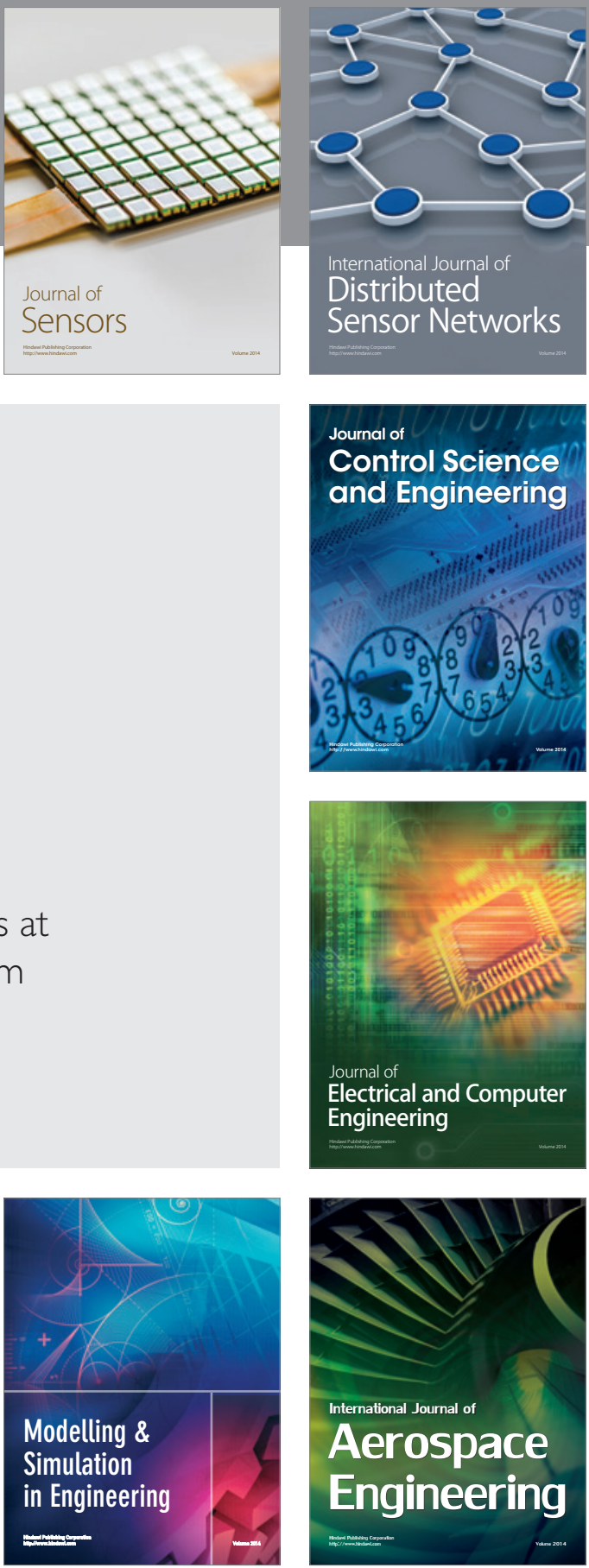

Journal of

Control Science

and Engineering
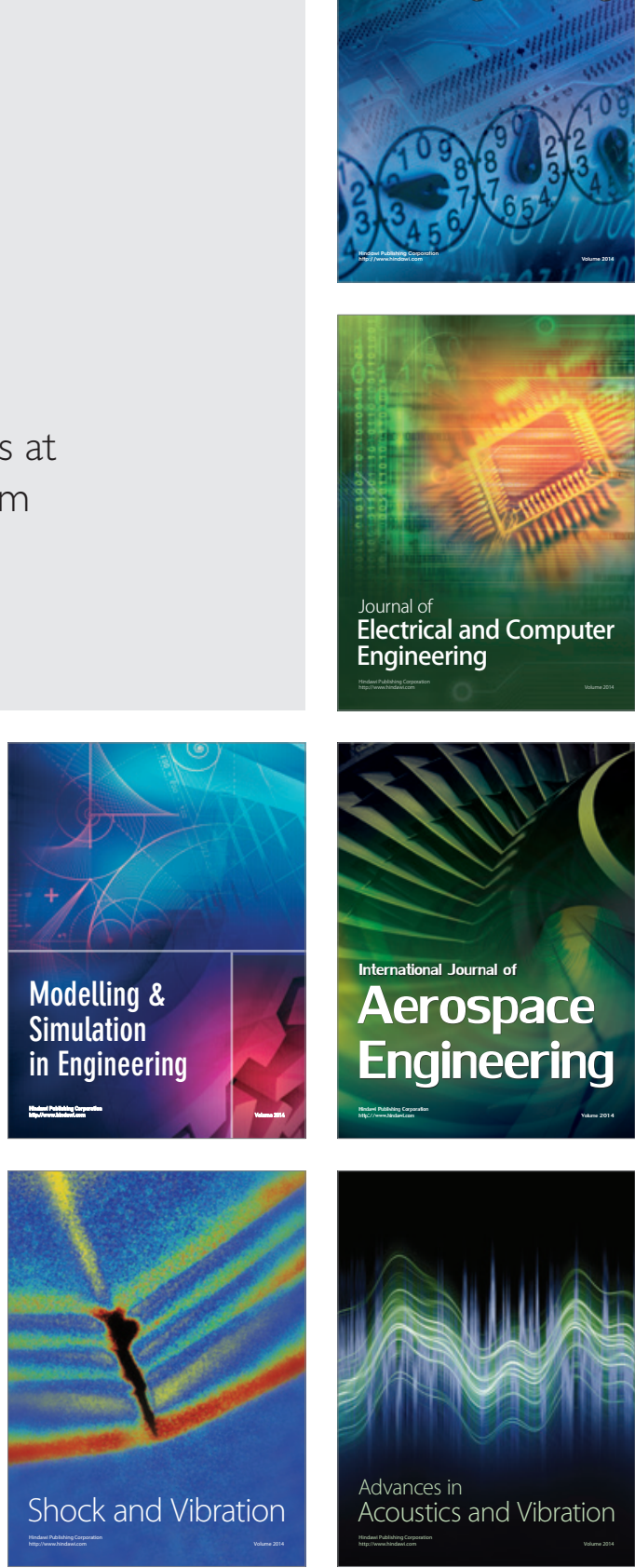